\title{
The Consultative Committee for Metrology in Chemistry and Biology - CCQM
}

\author{
Robert Kaarls $\odot^{*}$ \\ CCQM President, 1994 - 2013, Past Secretary CIPM, BIPM, France \\ (Received November 21, 2017; Revised December 15, 2017; Accepted December 20, 2017)
}

\begin{abstract}
Reliable, correct results of chemical measurements and analysis, with a measurement uncertainty statement fit for its purpose, are highly important for drawing the right conclusions and making the right decisions. Addressing the complex parameters influencing climate change, the consequences of unsafe food, the costly clinical diagnostics and the health effects of expensive pharmaceuticals, as well as addressing the need for new sustainable energy sources and fair trade, require accurate measurements. In 1993, by decision of the International Committee for Weights and Measures (CIPM), being the directive and supervisory body under the intergovernmental treaty, known as the "Metre Convention" and signed in 1875 with the aim of establishing and maintaining a global measurement system, the scientific Consultative Committee for Metrology in Chemistry and Biology (CCQM) was created. The CCQM is charged to establish global comparability of chemical and biological measurements and analysis through metrological traceability to the International System of Units, the SI, and very well defined pure reference materials and (primary) measurement methods and procedures. The article describes in short in which context the CCQM started and developed, how it is currently organized, what it has achieved and which priorities are being set in addressing the most important chemical and biological measurement issues in the near and medium term future.
\end{abstract}

Keywords: CCQM; BIPM; CIPM; metrology in chemistry and biology. (C) 2018 ACG Publications. All rights reserved.

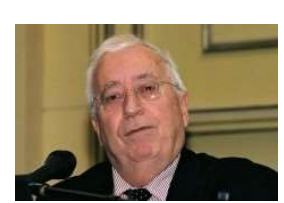

During the period 1994 - 2015 Robert Kaarls has been a member of the International Committee for Weights and Measures - CIPM. As such, from 1994 till 2013 he has been the President of the Consultative Committee for Amount of Substance - Metrology in Chemistry and Biology CCQM. From 2000 till April 2015 he has also been the Secretary of the CIPM. Formerly Robert Kaarls has been Director of the National Metrology Institute VSL of the Netherlands. He obtained his degrees in metrology, chemistry, electronic engineering and physics at the Delft Technical University. In 1967 he has been a guest worker at NBS (now NIST) in Washington DC and Robert Kaarls Gaithersburg, Md., USA

Robert Kaarls is a founding member, and has been the first chairman of EURACHEM. He is also a founding member of CITAC and the Joint Committee on Traceability in Laboratory Medicine - JCTLM. As Rapporteur of the CIPM Working Group on the Statement of Measurement Uncertainty he wrote in 1980 the first document giving guidance to nowadays measurement uncertainty calculations and statements as has been published in the Guide to the Expression of Uncertainty in Measurement - GUM. He has also been closely involved in the establishment and development of the CIPM MRA. During the period 1975 - 1999 Robert Kaarls has been involved in the organization and execution of laboratory accreditation. Among others, he has been a founding member and head of the Accreditation of Laboratories in the Netherlands and founding member and chairman of the Western European Calibration Cooperation - WECC and the European Accreditation of Laboratories - EAL, and founding member and vice-chairman of the European Accreditation - EA.

\footnotetext{
*E-mail: rkaarls@kpnmail.nl
}

The article was published by ACG Publications www.acgpubs.org/JCM $\odot$ January-June 2018 EISSN:1307-6183 DOI:http://doi.org/10.25135/icm.11.17.12.060 


\section{Introduction}

This paper describes the start of a coordinated global activity aiming to establish global comparability of (bio-)chemical measurement results through traceability to the International System of Units. The newly established Consultative Committee for Amount of Substance - CCQM, being a scientific advisory committee operating under the Inter-Governmental Treaty of the Metre Convention, has been charged with these activities solving the world-wide lack on comparability and metrological traceability. Starting with the insufficient metrological quality still existing in the domain of (bio-) chemical analysis/measurements in the second half of the $20^{\text {th }}$ century, the paper describes how a difficult start became within 25 years a very successful operation. Defining the essential differences between physical measurements and (bio-)chemical measurements, such as advanced technological developments and the discerned need to exactly identify the measurand and to describe precisely the measurement preparation procedure, as well as the excellent cooperation of all National Metrology Institutes and their stakeholders, have been key success factors. However, we are well aware that in the different fields of (bio-)chemical measurements still a lot of challenging work lies ahead of us.

\section{The History and the Need for Metrology in Chemistry}

Triggered by scientific considerations, rapid technical developments and the increasing needs of international trade, traffic and communication in the $19^{\text {th }}$ century, global cooperation aiming to realize a global unified and unique measurement system became an urgent issue. Therefore, 17 States from all around the world created in 1875 the International Bureau of Weights and Measures - BIPM, seated at the Pavillon de Breteuil in Sèvres, near Paris in France. The inter-governmental treaty, known as the "Metre Convention" and establishing the BIPM, was signed on 20 May 1875 by the governments of the 17 Member States. The treaty, and consequently the BIPM, originally focused in particular on mass (kilogram) and on length and volume (metre). Due to the industrial, technological and trade developments in the world, the $6^{\text {th }}$ General Conference on Weights and Measures (CGPM) of the Member States in its meeting in 1921 decided to modify the treaty by broadening it to cover also other physical measurement units and standards, and added electrical measurements and measurement standards, such as voltage, electrical resistance and current, to the BIPM activities.

During the following decades also temperature, radio activity and dosimetry, luminous intensity/photometry and time were added to the responsibilities of the BIPM. The $11^{\text {th }}$ General Conference of the Member States in 1960 formally adopted the International System of Units - SI, in those days comprising six base units, being the $\mathrm{kg}, \mathrm{m}, \mathrm{s}, \mathrm{A}, \mathrm{K}$ and $\mathrm{cd}$. Finally, proposed by the IUPAC, IUPAP and ISO, the $14^{\text {th }}$ General Conference in 1971 adopted the mole for amount of substance as the $7^{\text {th }}$ base unit to the SI. However, this did not immediately trigger any further chemical metrology oriented activities by the BIPM.

Although in some States the national metrology institutes already by the end of the $19^{\text {th }}$ century were charged with some chemical measurement activities, like the NBS (now NIST) in the USA, it is only in the late $60^{\text {th }}$, mid-seventies of the $20^{\text {th }}$ century, that it was felt that global coordination and comparability of chemical measurement results through metrological traceability will also be needed. For example, the VSL of the Netherlands noted problems with the international recognition and acceptance of its gas-mixtures, produced and certified by the VSL. Together with a few other organizations the BIPM was asked to consider starting coordination and carrying out some metrological activities in the field of chemical metrology. Unfortunately in those days the International Committee for Weights and Measures - CIPM, being the directive and supervisory board of the BIPM, elected by the Member States of the "Metre Convention", was not in a position, and could not be convinced, to start meaningful activities in the field of chemical metrology. 
However, due to the strongly increasing international trade of chemical products, World Trade Organization Technical Barriers to Trade (WTO TBT) measures and the development of accreditation systems for Certified Reference Material producers and chemical (test-)laboratories, and the subsequent wish of international recognition of these laboratories during the last decades of the $20^{\text {th }}$ century, all requiring internationally recognized metrological traceability, a number of national (chemical) metrology institutes approached again the BIPM and the CIPM with the request to reconsider its position concerning chemical metrology. Among these institutes were the LGC, in particular represented by Dr. Alex Williams, UK Government Chemist, and institutes such as NIST, VSL, and the IRMM.

So, in 1990 after more international chemical metrological coordination activities had been initiated, such as the creation of EURACHEM in 1989 and the signing of some bilateral agreements between a few NMIs, the CIPM decided to investigate the feasibility of meaningful chemical metrology activities by the BIPM together with the National Metrology Institutes (NMIs) of its Member States. Dr. John Lyons, being himself a chemist and in those days the Director of NIST and a member of the CIPM, was charged with organizing this feasibility study.

\section{The Start in $\mathbf{1 9 9 3}$}

The feasibility studies organized during the period 1990 - 1993 under the aegis of the CIPM included two comparisons of measurement results obtained by a number of NMIs having chemical metrology activities:

- The determination of $\mathrm{Pb}$ in water, led by NIST, USA. Isotope Dilution Mass Spectrometry was used for the analysis.

- The determination of $\mathrm{CO}, \mathrm{CO}_{2}$ and $\mathrm{NO}$ in nitrogen, led by NMi-VSL, Netherlands. Gas chromatography and non-dispersive infrared absorption were used for the analysis. The primary gas mixtures were prepared gravimetrically.

The first results of the measurement of heavy metals in water were, with uncertainties larger than the target uncertainty of $1 \%$, somewhat disappointing, demonstrating a lack of conformity in the measurement procedures used by the participating NMIs. It became clear that really all influence parameters in the measurement process had to be addressed, including the availability and purity assessment of the "pure" substances and calibration solutions used, as well as the details of the uncertainty budget calculations. So, this comparison had to be repeated by applying a much more clear and harmonized measurement procedure. And indeed, as reported in 1997, the results of the second comparison were much more satisfactory, all results now being within $1 \%$ of the gravimetrically determined reference value.
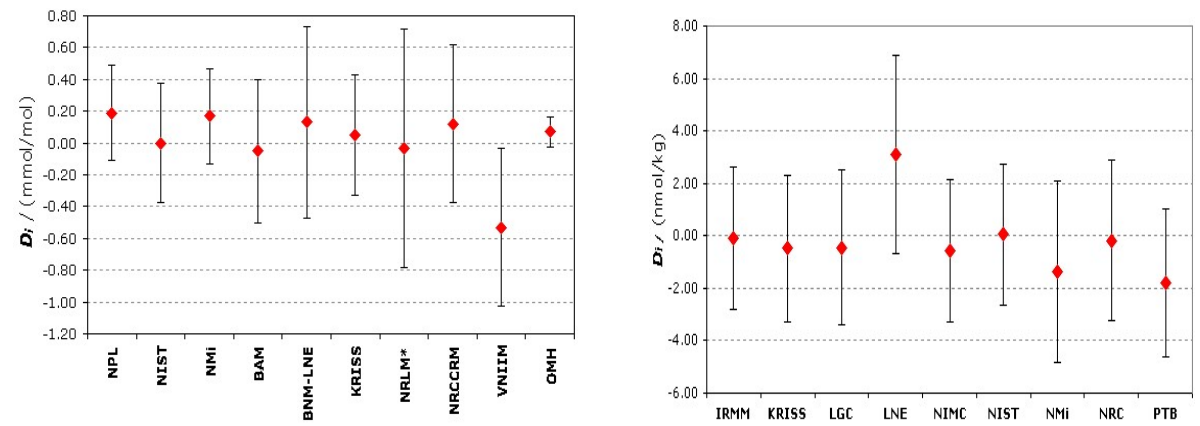

$\mathrm{CO}_{2}$ in $\mathrm{N}_{2} \quad 150 \mathrm{mmol} / \mathrm{mol}$

$\mathrm{Pb}$ in natural water nominal value $63 \mathrm{nmol} / \mathrm{kg}$

Degrees of Equivalence $D_{i}$ between participating institutes and expanded uncertainty $u_{i}(\mathrm{k}=2)$

Figure 1. The results of the first two Key Comparisons CCQM-K1.b and CCQM-K2 
The results of the measurement of the components in the gas mixtures were, with an uncertainty of mostly within $\pm 0,5 \%$, sufficiently acceptable, demonstrating that global comparability of measurement results, traceable to the SI, is feasible. The results of the first two Key Comparisons CCQM-K1.b and CCQM-K2 are presented below (Figure 1).

Based on the progress made and the results obtained from the two feasibility studies, the CIPM decided in 1993 to create a new Consultative Committee for Amount of Substance (metrology in chemistry) - CCQM, charged with:

- advising the CIPM on matters related to the accuracy of quantitative chemical measurements and traceability to the SI;

- coordinating the activities of national metrology laboratories in establishing traceability at the highest level;

- stimulating the understanding of the concept of uncertainty and the assignment of uncertainty statements in chemical measurements, thereby encouraging the establishment of traceability, taking into account other initiatives at regional and international levels;

- keeping under review the question of whether or not there is a need for a programme of work at the BIPM to support this activity.

In 1994 Dr. Robert Kaarls was appointed as the first President of the CCQM. He was assisted by the Executive Secretary of the CCQM, Dr. Richard Davis, and the BIPM Director, Dr. Terry Quinn (Figure 2).

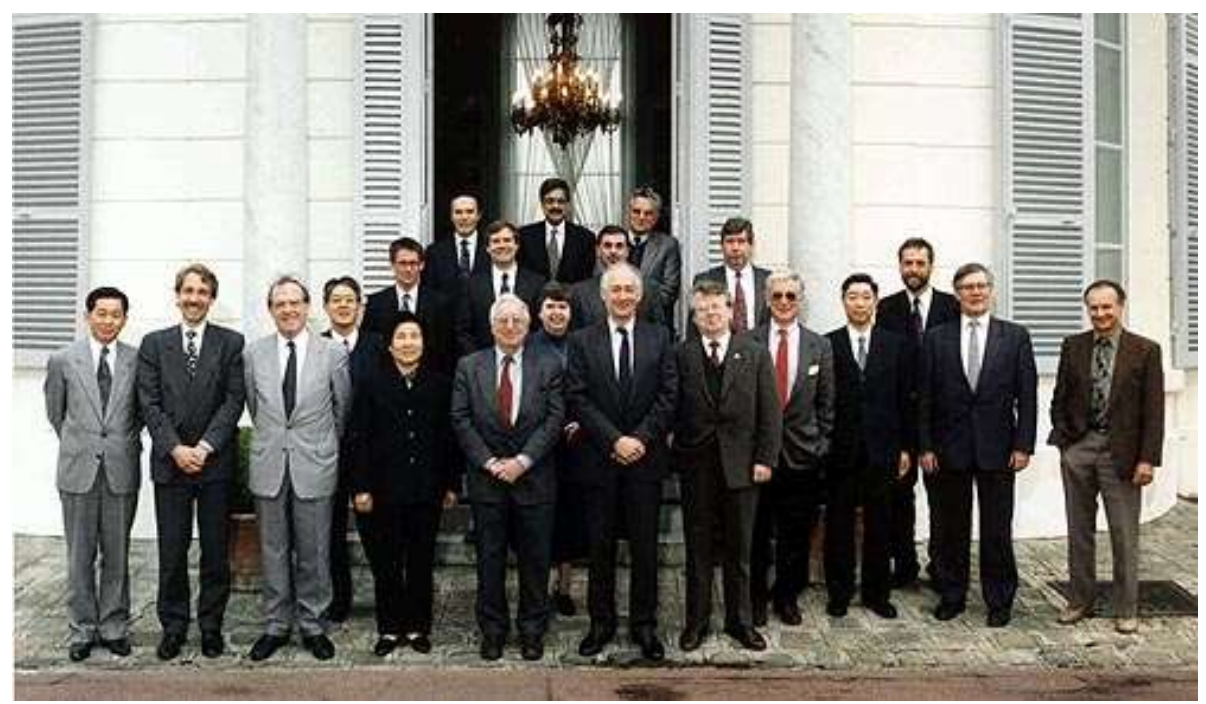

In February 1995 the first meeting of the CCQM was held at the BIPM attended by 20 representatives from 13 NMIs in 10 countries and 2 international organizations.

Figure 2. The first CCQM meeting

Apart of discussions on the results of the two feasibility studies, the major focus of the agenda was on chemical primary methods.

"A primary method of measurement is a method having the highest metrological qualities, whose operation can be completely described and understood, for which a complete uncertainty statement can be written down in terms of SI units, and whose results are accepted without reference to a standard of the quantity being measured.

A primary reference material is one having the highest metrological qualities and whose value is determined by means of a primary method."*

\footnotetext{
* (Note: over the years the exact wording of the definitions have been changed and adapted; see also the International Vocabulary of Basic and General Terms in Metrology - VIM)

Among others, potential primary methods could be gravimetry, freezing-point depression determination, differential scanning calorimetry, titrimetry, coulometry, IDMS, INAA, qNMR and different laser applications, some of them being a real primary "direct" method and others being in fact a "primary" ratio method.
} 
It is not only of importance to measure how much it is, but it is also essential to know, identify and define exactly what it is, one is measuring. The measurand/analyte has to be determined very clearly and in detail.

During the third meeting of the CCQM in 1997 it was decided to change the focus a little bit, and to start on a much broader basis with the organization of inorganic, organic and gas mixture comparisons. To run all the activities, the CCQM established during the period 1997 - 1998 four CCQM Working Groups on Gas Analysis, chaired by Mr Anton Alink (VSL), later on succeeded by Dr. Ed de Leer (VSL) and by Dr. Martin Milton (NPL), Inorganic Analysis, chaired by Mike Sargent (LGC), succeeding Dr. Bob Watters (NIST), Organic Analysis, chaired by Dr. Willie May (NIST), succeeding Dr. Bernard King (LGC), and Electro-chemical Analysis, chaired by Dr. Wolfgang Richter (PTB), later on succeeded by Dr. Michal Mariassy (SMU). In 2000 the CCQM established a fifth Working Group on Key Comparisons and CMC Quality, chaired by Dr. Hratch Semerjian (NIST), first succeeded by Dr. Jim McLaren (NRC) and later on by Dr. Lindsey Mackay (NMIA). The scope of the last Working Group being the coordination, harmonization and approval of Key Comparisons and, later on added, the final review of the Calibration and Measurement Capabilities (CMCs) claimed by the NMIs and other Designated Institutes (DIs).

The first four CCQM Working Groups started with organizing Pilot Study Comparisons and Key Comparisons of rather simple and not too complex chemical matrixes, and from there moving to the more complex materials. This approach has demonstrated to be very successful. Examples are $\mathrm{Cd}$ and $\mathrm{Pb}$ in water, $\mathrm{pp}$ '-DDE in iso-octane and cholesterol in serum. Of course, it was well understood that traceability to the SI had to be part of the exercise, so all used measurement methods had to be traceable to a method of "higher metrological order", and to traceable, well characterized calibration solutions and pure primary reference materials.

The CCQM also discussed future work in support of chemical surface measurements/analysis and work in support of clinical chemistry and biotechnology. Starting as an ad hoc Working Group, the CCQM established the CCQM WG on Surface Analysis in 2000, chaired by Dr. Martin Seah, later on succeeded by Dr. Wolfgang Unger (PTB). Likewise, starting as an ad hoc Working Group the CCQM established the CCQM WG on Bio Analysis in 2001, co-chaired by Dr. Gary Gilliland (NIST) and Dr. Helen Parkes (LGC), later on chaired by Dr. Helen Parkes.

Discussions on whether the BIPM should have also itself a chemical metrology laboratory concluded that the BIPM, in order to be able to represent and speak on behalf of the global metrological community in this new field with authority, must have itself the necessary knowledge and experience in chemical and biological measurements/analysis. Therefore, the CIPM decided in 1998 that the BIPM should establish a limited programme of metrology in chemistry, starting in the field of gas analysis by coordinating comparisons and improving the accuracy. It was also decided that the BIPM in close cooperation with NIST should have a primary ozone standard reference photometer. Meanwhile this primary reference photometer has been improved considerably by additional studies on the absorption cross-section of ozone, carried out by the BIPM staff.

Proposed by the CCQM, the CIPM decided in 2003 to broaden the scope of the BIPM activities by developing capabilities for purity analysis of larger organic molecules, to be used as primary calibrators for clinical and organic chemistry. These primary calibrators play a key role in establishing traceability in the field of clinical and organic analysis/measurements.

A questionnaire sent to all NMIs concerned showed that there is a high need for these calibrators. It also showed that almost none of the NMIs and DIs are producing these calibrators. So, a commonly paid capability at the BIPM serving all NMIs and DIs is considered to be an efficient and effective investment, not duplicating work already carried out by the NMIs and DIs, but having essential added value to the activities carried out by the NMIs and DIs.

Also the second meeting in 1996 mainly focused on what are the primary methods, how useful are these and how do they underpin or deliver SI traceability to the generally used analytical analysis/measurements methods used by the chemical measurement and testing laboratory community. In the field of gas mixtures there is a straight way forward, but for inorganic and organic analysis this is much less clear. Moreover, in several cases the repeatability of inorganic and organic analysis seems to be much better than the measurement uncertainties obtained when the traceability chain is followed starting with a primary method. Also it had to be understood that purity analysis, needed to produce very pure reference materials, is an essential step in the chemical metrology hierarchy.

In fact, and that is different from the physical measurement field, the top of the hierarchy in chemical metrology is a primary method and a well-defined and identified primary pure material (pure certified reference material). 


\section{Development and Achievements}

Triggered by the needs of global trade, the WTO and its Technical Barriers to Trade policy, accreditation organizations and regulators, the CIPM established in 1999 the CIPM Mutual Recognition Agreement - CIPM MRA, on 14 October 1999 for the first time signed by many NMI Directors. Currently the CIPM MRA has been signed by the NMI Directors, representing the NMIs and other Designated Institutes (DIs) from 98 Member States and Associates (States) of the CGPM, and 4 international organizations, being the IAEA, WMO, EU JRC and the ESA. Under this CIPM MRA the national measurement standards, calibration and measurement capabilities, produced Certified Reference Materials and the Calibration and Measurement Certificates issued, which fulfil well defined criteria, and are regularly delivered to the customers of the NMIs and the other DIs, can become globally recognized and accepted. Criteria to be fulfilled are clear traceability to the SI, or if not yet feasible to other internationally agreed references, regular participation in the activities of the relevant Working Groups of the CIPM Consultative Committees, among these the CCQM, and/or Regional Metrology Organizations (RMOs), and the comparisons organized by these WGs and RMOs. Further one has to comply fully with the relevant quality assurance criteria defined in the current ISO 17025, and additional rules defined by the CIPM and CCQM with respect to the CIPM MRA. The creation of the CIPM MRA has given again a firm boost to the further development of metrology in chemistry and biology.

Meanwhile in 2003, ten years after its creation, the CCQM meeting was attended by some 55 representatives, representing 32 institutes from 24 States and 6 international institutes (IAEA, EUIRMM, IFCC, IUPAC, ISO REMCO, ILAC). The participation of the chemical metrology experts in the CCQM WGs is a multiple of the attendance of the CCQM plenary. And every year more NMIs and DIs are attending and joining in the CCQM activities.

The creation of the CIPM MRA has urged further and intense discussions on what is, and how to calculate, a Key Comparison Reference Value (KCRV), and how to calculate the different Degrees of Equivalence (DoEs) between the participating NMIs and DIs, and the related measurement uncertainty. And what are the criteria for acceptance of a claimed Calibration and Measurement Capability (CMC) for publication in the Key Comparison Data Base (KCDB) of the CIPM MRA.

The rapidly growing interest of the NMIs and DIs in metrology in chemistry and biology, and the wish to participate in the CIPM MRA has led to a strongly increased number of CCQM comparisons. The way to organize and describe comparisons had to become much more formal in order to be a firm basis for obtaining meaningful and useful results.

Based on the results of comparisons already carried out before the establishment of the CIPM MRA, the participating NMIs and DIs started to publish their approved CMCs in the KCDB.

In order to have, as much as possible, a clear Data Base accessible and understandable for the external user, the CCQM created 15 categories, comprising all the deliverables, being the CMCs of the NMIs and DIs.

In the table below these categories are presented with the number of CMCs published in the KCDB, as well as the number of countries having declared CMCs by the end of December 2016 (Table 1). 
Kaarls, J. Chem.Metrol. 12:1 (2018) 1-16

Table 1. Declared CMCs by the end of December 2016 in KCDB

\begin{tabular}{|c|c|c|}
\hline Category & CMCs & Countries \\
\hline High purity chemicals & 656 & 17 \\
\hline Inorganic solutions & 409 & 19 \\
\hline Organic solutions & 498 & 16 \\
\hline Gases & 2340 & 32 \\
\hline Water & 224 & 20 \\
\hline $\mathrm{pH}$ & 92 & 21 \\
\hline Electrolytic conductivity & 45 & 15 \\
\hline Metal and metal alloys & 226 & 13 \\
\hline Advanced materials & 144 & 14 \\
\hline Biological fluids and materials & 399 & 18 \\
\hline Food & 502 & 25 \\
\hline Fuels & 61 & 10 \\
\hline Sediments, soils, ores and particulates & 562 & 16 \\
\hline Other materials & 61 & 11 \\
\hline Surfaces, films and engineered materials & 8 & 4 \\
\hline Total CMCs & 6277 & \\
\hline
\end{tabular}

Triggered by a new EU Directive on In Vitro Diagnostics, requiring metrological traceability for clinical measurements, and following a workshop on "Measurement Traceability for Clinical Laboratory Testing and In Vitro Diagnostic Test Systems", held at NIST in the autumn of 2000, the CCQM, BIPM and the IFCC met a number of times, discussing the possibilities for improving the quality, reliability and metrological traceability of In Vitro Diagnostic clinical analysis by bringing together the experts of the clinical chemistry and the chemical metrology communities. Related discussions took place with a number of interested NMIs, the BIPM, CCQM, IFCC, ILAC and the WHO in Geneva, and one of its major reference laboratories, the National Institute for Biological Standards and Control (NIBSC) in the UK, with the aim of creating a database listing the approved reference materials and reference measurement procedures, as well as a network of reference laboratories in laboratory medicine, all fulfilling criteria defined by relevant ISO standards, IFCC, and metrological rules and legal regulations.

As a result in 2002 the Joint Committee on Traceability in Laboratory Medicine - JCTLM was established with the mission to support the worldwide comparability, reliability and equivalence of measurement results in laboratory medicine. This in its turn triggered several new comparisons in the field of clinical chemistry, for example cholesterol in human serum and creatinine in serum. The work on purity analysis, such as on steroid hormones, is another example in support of the work of the JCTLM.

Since 2004 the CCQM and its WGs have organized a large series of workshops, many of them together with the experts of our stakeholder organizations. Among others, workshops have been held on:

- Higher Order Measurement Methods for Physiological Significant Molecules,

- Pharma and Bio-Pharma Measurements with the pharmaceutical industry,

- The Frontiers of Traceability in Chem/Bio Measurements,

- Clinical Chemistry with the IFCC and the WHO,

- Protein and Peptide Therapeutics and diagnostics,

- Food Safety with Codex Alimentarius Commission of the WHO and FAO,

- Reliable Traceable Microbiological Measurements to Ensure Food Quality and Safety with the food safety authorities such as the US FDA, and food testing laboratories and food industry, 
- Measurements of the Atmosphere and of Ambient Air Quality and Standards and Measurements for Clean Air with the WMO and its Global Atmospheric Watch project,

- Global to Urban Scale Carbon Measurements with authorities such as the EPA,

- Forensics with US DEA, Japan Police authorities and forensic laboratories,

- Standards and Metrology in Support of Anti-Doping Analysis with the WADA,

- Certified Reference Material for Quality of Life with CRM producers and standardizers such as ISO REMCO,

- Carbon Dioxide and Methane Stable Isotope Gas Standards with the International Atomic Energy Agency (IAEA) and the International Union of Pure and Applied Chemistry.

In many of these workshops also the ILAC participated, inasmuch as traceable measurements with an accuracy fit for its purpose are in fact a major requirement in the ISO 17025 standard, being the basis for laboratory accreditation.

The cooperation between the CCQM WG on Gas Analysis with the WMO Global Atmospheric Watch (GAW) group became very intense, strengthening the cooperation with a number of (inter)national meteorological organizations in several countries around the world, like NOAA and the World Calibration Centre and Central Calibration Laboratory activities in the scope of the WMO- GAW. The further improvement of the ozone standard by the BIPM and NIST has largely contributed to much more accurate ozone measurements.

A very active contribution in establishing and improving the relations with the WMO GAW has been delivered by Dr. Martin Milton (before at NPL, UK and chairman of the CCQM GAWG from 2008 through 2012), and currently Director of the BIPM.

A workshop on Measurements Challenges for Global Observation Systems for Climate Change: Monitoring Traceability, Stability and Uncertainty, organized in 2010 by the BIPM and the WMO and held in Geneva, has given a further boost to the improvement of climate change measurements, not only covering gas analysis, but for example also improving the measurement of the conductivity of seawater, as an important indicator for climate change and tsunami warning systems, and improving the reliability and coherence of airborne and satellite observations.

The inter-disciplinary cooperation with the experts of our stakeholders has very much contributed to an increased awareness of the importance of accurate and traceable measurement and test results, and has given the CCQM great guidance in setting the right priorities and creating added value by solving specific metrological problems in the fields concerned.

Over the last decade metrology in (bio-)chemistry activities has also been taken on board of the Regional Metrology Organizations (RMOs), multiplying the CCQM activities in the region, helping newcomers in the region to start up their own (bio-)chemical activities, and on a regional basis organizing awareness and training courses, as well as in a number of cases involving the regional accreditation bodies by delivering reference values to Proficiency Testing (PT) schemes. In this way increased awareness about the usefulness and efficacy of traceability is communicated and transferred to a large number of chemical testing laboratories.

Under the leadership of Dr. Robert Wielgosz, Director of the Chemical Department of the BIPM, the (bio-)chemical metrology activities at the BIPM developed very quickly and started to make important contributions to the activities carried out under the aegis of the CCQM, in particular focusing on the ozone standard, gas mixtures comparisons and purity determinations of organic molecules. As mentioned before, the crucial role of completely identified and defined, high accuracy and stable primary pure reference materials as basic cornerstones in establishing metrological traceability in (bio-)chemical measurements and analysis is well understood. It is also therefore that the Organic Analysis group of the BIPM Chemistry Department has a unique focus on the purity analysis of small and larger complex molecules, such as digoxin, estradiol, aldrin, L-valine, bisphenol and peptides. 
Examples of the BIPM work, such as the results of ozone in air (BIPM.QM-K1) and the results of the purity assessment of a high purity organic material aldrin (CCQM-K55.b) are given below (Figure $3)$.
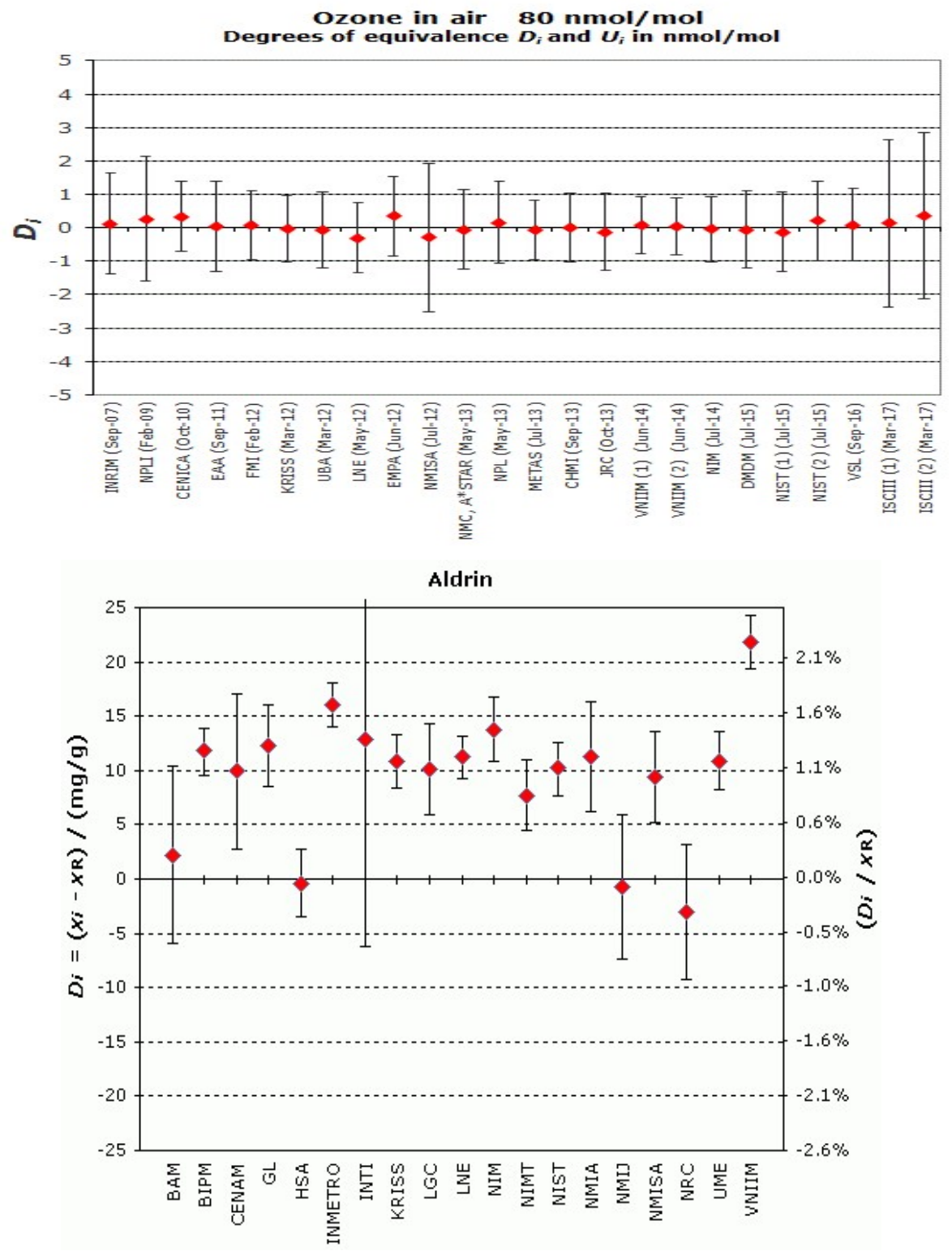

Degrees of equivalence: offset $D_{i}$ and expanded uncertainty $(k=2) U_{i}$ expressed in $\mathrm{mg} / \mathrm{g}$, and also in relative terms, $X_{R}=950.8 \mathrm{mg} / \mathrm{g}$ and $2 U_{\mathrm{R}}=1.70 \mathrm{mg} / \mathrm{g}$

Figure 3. Examples of the BIPM works, ozone in air (BIPM.QM-K1) and high purity organic material Aldrin (CCQM-K55.b) 


\section{Current CCQM organization and activities}

In 2000 surface analysis (by chemical analysis) and in 2001 bio analysis were added to the scope of activities of the CCQM.

Although being a more specialized field, surface analysis is a very important activity in for example the advanced materials industry, the electronic chip industry, the aircraft and space industry and increasingly also in the medical and biological area. Comparisons carried out include comparisons and understanding of the characteristics of different types of surface analysis techniques and methods, such as $\mathrm{SiO}_{2}$ on $\mathrm{Si}$ film thickness, quantitative analysis of $\mathrm{Fe}-\mathrm{Ni}$ alloy, electron probe micro analysis on $\mathrm{Au}-$ $\mathrm{Cu}$ alloys and measurement of porosity properties.

With respect to biological measurements, like during the first years of the CCQM starting to work itself in 1994, the question is not whether there is a need to improve the quality of biological measurements, but how to tackle the problem and what to address first. So, after a period of study and the organization of a few first comparisons, such as quantitative PCR, activity of alpha amylase in human serum, relative quantification of genomic DNA fragments extracted from a biological tissue, and relative quantification of Bt63 in GM rice matrix, the activities of the CCQM Working Group on Bio Analysis (BAWG) developed well and arrived at a level of specialization, which demanded a more focused organization. A first example of the work by the BAWG is given below (Figure 4).

\section{MON810/hmg copy number ratio}

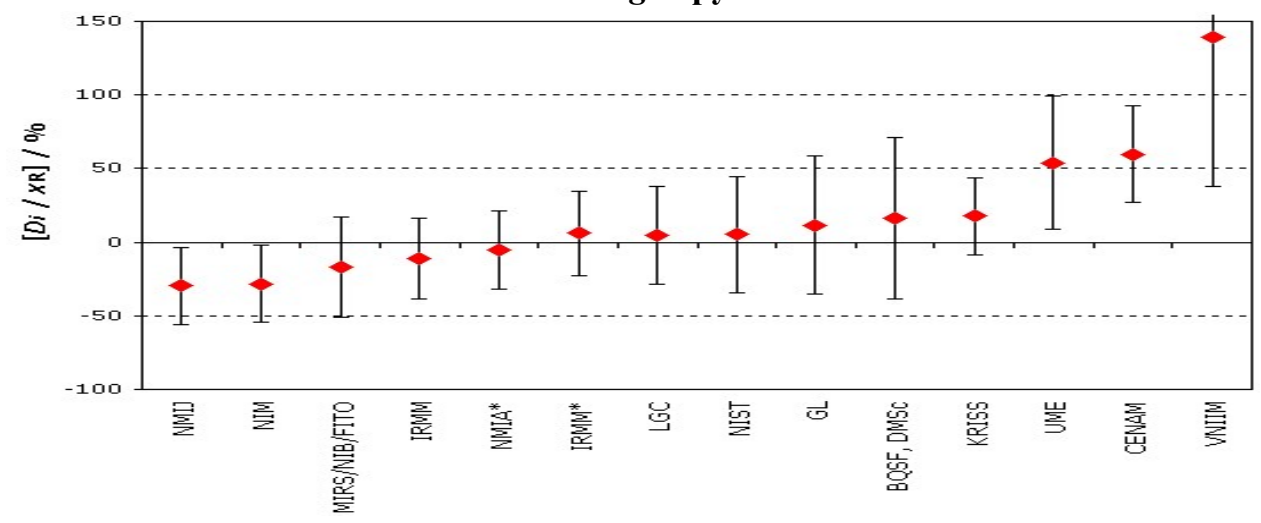

Degrees of equivalence: $D_{i}$ and expanded uncertainty $U_{i}(k=2)$ given in relative terms

Figure 4. Relative quantification of genomic DNA fragments extraxcted from a biological tissue (CCQM-K86)

In 2011, after the CCQM had organized an interesting workshop on the Role for Reliable Traceable Microbiological Measurements to Ensure Food Quality and Safety, an ad hoc CCQM Steering Group on Microbial Measurements (MBSG) was set up, chaired by Dr. Lauri Locascio (NIST), who was later on succeeded by Dr. Jane Morrow (NIST). The workshop was attended by 45 participants representing a wide spectrum of stakeholders active in biological measurements, among these the FDA, USDA, EU-RL, APEC, AOAC, ISO/CEN, CRM producers ATCC, IRMM, LGC, PT providers, International Diary Federation, major multinational food industries, food testing laboratories, food testing kit manufacturers and NMIs. The workshop discussed current measurement problems/issues relating to sampling, cell/organism growth, colony count, detection, isolation, identification, characterization, reference methodologies and assay techniques for the assessment of pathogens, such as bacteria, viruses, fungi, moulds, yeast. Problems associated with ill-defined measurands, unsound metrological reference methods, insufficient global harmonization, lack of calibration hierarchy and a 
lack of CRMs were identified. The MBSG started with two projects, the first being a microbial identity investigative study, aimed to establish comparability between laboratories measuring 16S rRNA sequence. The second project aimed to establish comparability for cell counts by the plate count method for Listeria monocytogenes, which is a food safety and public health relevant organism.

The interest in the activities of the CCQM Bio Analysis Working Group and in the Microbial Measurements Steering Group is demonstrated by the participation in the meetings and their study projects and Pilot Study and Key Comparisons of an increasing number of experts from a growing number of NMIs, DIs and other stakeholder organizations, all having increasing activities in this field.

As a consequence of the much broader tasks of the CCQM the name and the scope of the CCQM were officially broadened in 2014 to "CCQM - Metrology in Chemistry and Biology".

Also, as a consequence of the increased and more specialized participation, in 2014 the CCQM decided to split the BAWG in three new CCQM Working Groups, being the Cell Analysis WG - CAWG, Protein Analysis WG - PAWG and the Nucleic Acid WG - NAWG, and integrate the activities of the MBSG in these three new CCQM WGs.

The CCQM with its participating NMIs and DIs now serves in close cooperation with its stakeholders a very wide scope of customers.

Cooperation with the health sector has increased, not only through the already existing cooperation with the JCTLM, but also in particular by the participation in the CCQM of the NIBSC, a UK DI and a major WHO laboratory.

The cooperation with the World Anti-Doping Agency - WADA has been further strengthened after the BIPM-WADA Symposium in 2016.

Likewise the already existing cooperation with the WHO GAW and its calibration and reference laboratories has been further intensified.

The CCQM is now the largest CIPM Consultative Committee.

The CCQM is responsible for developing, improving and documenting the equivalence of national standards (certified reference materials and reference methods) for chemical and biological measurements. It advises the CIPM on matters related to chemical and biological measurements including advice on the activities carried out in the scope of the BIPM scientific programme.

Consequently the CCQM is charged with:

a. establishing global comparability of measurement results through promoting traceability to the SI, and where traceability to the SI is not yet feasible, to other internationally agreed references;

b. contributing to the establishment of a globally recognized system of national measurement standards, methods and facilities for chemical and biological measurements;

c. contributing to the implementation and maintenance of the CIPM MRA with respect to chemical and biological measurements;

d. reviewing and advising the CIPM on the uncertainties of the BIPM's calibration and measurement services as published on the BIPM website;

e. acting as a forum for the exchange of information about the research and measurement service delivery programmes and other technical activities of the CCQM members and observers, thereby creating new opportunities for collaboration.

The official membership of the CCQM has grown to 25 member organizations, 10 observer organizations and 6 Liaison organizations, including the IUPAC, IAEA, ISO REMCO, IFCC, EU JRCGeel (IRMM) and CITAC, while ILAC is still indirectly involved. The plenary meeting is further attended by invited guests from major stakeholder organizations and from upcoming, new NMIs and DIs. 
In the yearly meetings of the CCQM and its working groups in April at the BIPM more than 250 experts participate, while some of the major CCQM WGs also have a meeting in the second half of the year, generally housed by one of the participating NMIs or DIs. Running part of the CCQM WG meetings at the premises of the participating NMIs and DIs also contributes considerably to increased mutual confidence and an intensified exchange of knowledge and experience, while also creating wider awareness through additional symposia, organized by and in the hosting country.

Since CCQM's start in 1993, now in 2017 there are registered in the KCDB database of the CIPM MRA well more than 150 CCQM Key Comparisons and well more than 200 CCQM Pilot Study Comparisons and some 40 chemical RMO comparisons. The results of these comparisons are underpinning about 6227 approved CMCs, published in the KCDB.

The CCQM (Figure 5), has currently 10 standing Working Groups and 2 ad-hoc Working Groups. Its organization is:

- CCQM President Dr. Willie E. May (CIPM)

- CCQM Executive Secretary Dr. Robert Wielgosz (BIPM)

- Gas Analysis (GAWG), Chairperson Dr. Jin Seog Kim (KRISS)

- Inorganic Analysis (IAWG), Chairperson Dr. Mike Sargent (LGC)

- Organic Analysis (OAWG), Chairperson Dr. Lindsey Mackay (NMIA)

- Electrochemical Analysis (EAWG), Chairperson Dr. Michal Mariassy (SMU)

- Surface Analysis (SAWG), Chairperson Dr. Wolfgang Unger (BAM)

- Nucleic Analysis (NAWG), Chairperson Dr. Helen Parkes (LGC)

- Protein Analysis (PAWG), Chairperson Dr. Sang-Ryoul Park (KRISS)

- Cell Analysis (CAWG), Chairperson Dr. Jane Morrow (NIST)

- Key Comparison and CMC Quality (KCWG), Chairperson Dr. Della Sin, (HKGL)

- Strategic Planning (SPWG), Chairperson Dr. Willie May (CIPM)

- Ad-hoc Working Group on the Mole, Chairperson Dr. Bernd Güttler (PTB)

- Ad-hoc Working Group on the KCDB 2.0, Chairperson Dr. Della Sin

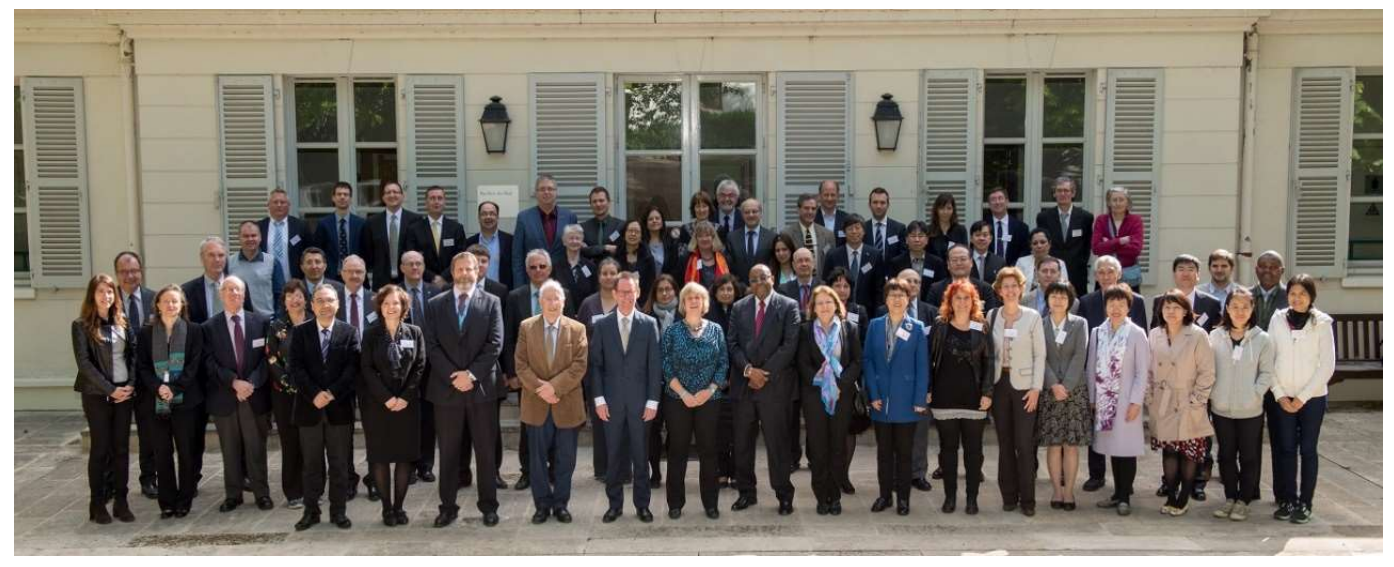

Figure 5. The $23^{\text {st }} \mathrm{CCQM}$ meeting in 2017 at the BIPM in Sèvres, France

\section{Future Focus}

As the scope of the CCQM activities is very wide and the measurement ranges of all analytes and the number of chemical and biological matrixes is endless, the CCQM has initiated a process of reducing the costs of comparisons by restricting the number of comparisons to be carried out. This is done by developing a system where only the most essential comparisons, giving a wide overview of the "core" capabilities and competences of the participating NMIs and DIs, are carried out. This 
type of comparisons, to be repeated for example once every two to four years or reproduced by a different comparison, but addressing the same laboratory capabilities and competences, should also answer a major question, already in the beginning tabled by the chemical metrology community, namely "How far does the light shine?" Or in other words: how many, preferably broader based, CMCs are covered by the "light" of the results of a single Key Comparison.

In principle one can distinguish between 4 types of comparisons (Figure 6).

A. Comparisons designed to test core capabilities, skills and competences. As an example of the approach in the organic analysis field can be mentioned that one has agreed to plan 20 comparisons, covering both primary calibrators as well as complex matrix reference measurements, underpinning CMCs across all core organic measurement capabilities The high-purity organic measurements space can be divided in 3 parts of molecular weight and polarity. By selecting a limited number of pure materials one can reduce the amount of comparisons to be carried out, while still covering the whole area of organic purity analysis capabilities. The so called track A comparisons may also include the comparison of the deliverables of the NMIs and DIs, such as Certified Reference Materials and calibration solutions.

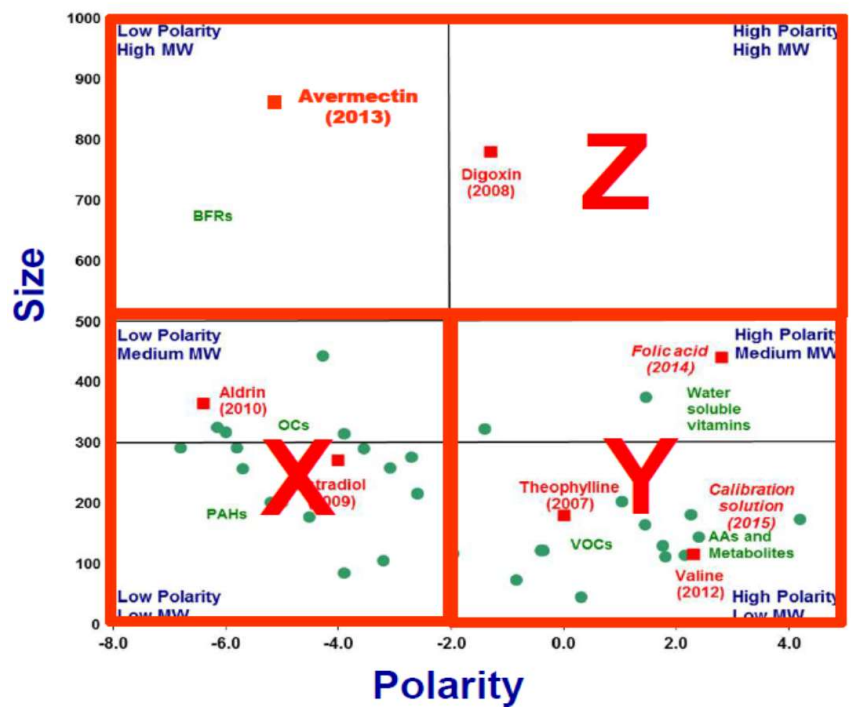

Figure 6. Design of CCQM comparisons based on the molecular weight and polarity

B. Comparisons designed to support CMC claims for components which present analytical challenges, and which consequently demand more specialized skills and competences than required for the capabilities tested by A.

C. Comparisons designed to test fully new, future CMCs, for example in emerging areas.

D. Comparisons, often Pilot Study Comparisons, for studies, learning, try-outs, etc. These comparisons are not intended to be used for underpinning CMCs published in the KCDB.

As part of the approach mentioned above it is the intention to publish in the KCDB, whenever possible and meaningful, CMCs with a wider, broader scope.

Connecting Proficiency Testing (PT) schemes, organized by a local NMI, DI or RMO, to a CCQM comparison is also fostered. In general one can conclude that PT schemes with a traceable reference value are of high value to the testing laboratories participating in the PT scheme, giving extra information with respect to the accuracy of their measurement/analysis activities.

In support of a sustainable economy, innovation, regulation and societal well-being, the CCQM activities will focus in particular on measurement sectors such as health care, food safety and nutritional 
value, environment, climate change, energy, manufacturing and advanced materials. Although in the more "classical" areas of chemical metrology very good progress has been made in establishing and delivering metrological traceability, still work has to be done in the areas of the more challenging reactive, complex and large molecules. Examples are medical gases, point of care in vitro diagnostics, pharmaceuticals, nanoparticles for diagnostics and drugs, contaminants in food, including mycotoxins and seafood, $\mathrm{pH}$ and salinity of the oceans, isotope ratio measurements, water quality, energy from bio waste, sustainable microbial energy, organic and inorganic thin film technologies, nano-particles, etc..

And, of course, in the biology oriented working groups still a lot of development is underway, such as DNA, RNA, GMO, nucleic acid sequencing, regenerative medicines, peptides, proteins, cells, eukaryotic cell refence materials, biological drugs, etc.. Also the hindering lack of higher order reference methods and materials in bio-measurements will need to be addressed.

Further development of accurate measurement techniques will be fostered, for example in surface chemical analysis at the micro scale, 3D Raman microscopy, aerosol measurements, spectroscopic methods, sampling for quantitative analysis and quantitative elemental imaging, etc..

The very useful inter-disciplinary cooperation with other international stakeholder organizations will be continued, for example with the IAEA on isotope ratio standards.

The activities of the BIPM Chemistry Department will continue to focus on improving the ozone standard, on coordinating comparisons for primary calibrators for prioritized greenhouse gases and air quality gases, and carrying out purity assessment for primary reference materials for small and large organic molecules. Further, the BIPM programme of work includes capacity building projects for support training and skills broadening secondments. All BIPM activities are rather unique and supplementary to the activities carried out by the NMIs and DIs.

\section{Redefinition of the Mole}

Depending on the use, results of (bio-)chemical measurements/analysis are expressed in different SI units, such as $\mathrm{mol} / \mathrm{mol}, \mathrm{mol} / \mathrm{kg}, \mathrm{mol} / \mathrm{l}, \mathrm{mg} / \mathrm{g}$, and $\mathrm{nm}$ (in surface analysis), or eventually non-SI units, such $\mathrm{pH}$, copy number and cell counts.

One of the tasks of the CCQM (French acronym for Consultative Committee for Amount of Substance) is also to advise the CIPM with respect to the need, use and definition of the measurement units, and in particular concerning the definition of the base unit mol.

Scientific and technological developments over the recent decades have made it possible to redefine almost all the base units of the SI in terms of the fundamental constants of nature. As a consequence, many other measurement units and constants can be defined with a (much) smaller measurement uncertainty than before.

So, the $26^{\text {th }}$ CGPM in 2018 will be proposed to redefine also the mole, symbol mol, as the SI unit of amount of substance in terms of the Avogadro constant, its magnitude to be set by fixing the numerical value of the Avogadro constant $N_{\mathrm{A}}$ to be $6.022140857 \times 10^{23}$ when expressed in the SI unit $\mathrm{mol}^{-1}$. (Note: the final value of the last digits to be set by the CODATA Task Group on Fundamental Constants prior to the $26^{\text {th }}$ CGPM in 2018)

The number of elementary entities, which may be atoms, molecules, ions, electrons, any other particles or specified group of particles, in one mole is then equal to the numerical value of the Avogadro constant, which is known as the Avogadro number. As a consequence of the new definition of the mole, the Avogadro constant and the Avogadro number will have no uncertainty.

This will replace the 1971 definition of the mole, defining the mole as "the amount of substance of a system which contains as many elementary entities as there are atoms in 0.012 kilogram of carbon 12 ". This means that the atomic mass constant $m_{\mathrm{u}}$ and the molar mass constant $M_{\mathrm{u}}$ will now get a very 
small experimental uncertainty. However, this uncertainty is so small that it will not have any effect on the traceability and measurement uncertainty of all chemical and biological analysis/measurements nowadays made.

\section{Conclusion}

In 2018 the CCQM exists 25 years and in 2019 it will have its $25^{\text {th }}$ plenary CCQM meeting. Enormous progress has been made in establishing global comparability through metrological traceability with a very much reduced measurement uncertainty in the field of chemistry and biology.

Under the current CCQM Presidency of Dr. Willie May, Past Director of NIST, USA, assisted by the CCQM Executive Secretary Dr. Robert Wielgosz, CCQM rapporteurs and all the excellent CCQM Working Group Chairpersons, in the past and currently, the CCQM will be able to continue its activities, thanks to the investments and expert scientists of the participating NMIs and DIs.

The results underpin a reliable, high quality CIPM MRA with a KCDB filled with reliable useful information for all types of users of this database. Work is ongoing to improve the quality and usefulness of this database.

\section{Acknowledgement}

The author likes to thank all NMIs, DIs and stakeholder organizations for their great and open minded support to the development of metrology in chemistry and biology, and in particular for their support to the CCQM.

The author also recognizes all the support and contributions of the experts from these organizations, participating in the CCQM plenary meetings, working group meetings and workshops, and their support as rapporteur of the CCQM plenary or rapporteur/secretary to the CCQM Working Groups

ORCID

Robert Kaarls: $\underline{0000-0001-7555-0718}$

\section{References}

[1] Convention du Mètre, signée 20 Mai 1875 et Règlement Annexé signee 6 Octobre,1921, BIPM

[2] From Artefacts to Atoms, Terry Quinn, Oxford University Press, 2012

[3] A.Williams (1999). EURACHEM - the first ten years, Alex Williams,; Eurachem - History and Impact, https://www.eurachem.org/images/stories/about/pdf/history-booklet.pdf

[4] International Vocabulary of Metrology - Basic and General Concepts and Associated Terms (VIM), $3^{\text {rd }}$ edition, JCGM 200:2012 (E/F), www.bipm.org

[5] Consultative Committee for Amount of Substance: Metrology in Chemistry (CCQM), Reports of the $1^{\text {st }}$ meeting in 1995 through the $20^{\text {th }}$ meeting in 2014, CIPM

[6] Consultative Committee for Amount of Substance: Metrology in Chemistry and Biology (CCQM), Reports of the $21^{\text {st }}$ meeting in 2015 through the $23^{\text {rd }}$ meeting in 2017, CIPM

[7] Mutual recognition of national measurement standards and of calibration and measurement certificates issued by national metrology institutes, Paris, 14 October 1999, CIPM-BIPM

[8] Declaration of Cooperation between the BIPM, IFCC and ILAC, for the Establishment of a Joint Committee for Traceability in Laboratory Medicine (JCTLM), www.bipm.org/en/committees/jc/jctlm; www.bipm.org/jctlm;

[9] CIPM MRA Data Base KCDB, www.kcdb.bipm.org; www.kcdb.bipm.org/appendixC/default.asp

[10] CCQM Strategy Document for Rolling Programme Development, period 2013-2023, www.bipm.org/en/committees/cc/ccqm/strategy.html; 
[11] Planned Future CCQM Comparisons (as of $1^{\text {st }}$ December 2016), BIPM - CCQM Strategy, www.bipm.org [12] CCQM Strategy Document for Rolling Programme Development, period 2017-2026, internal draft document

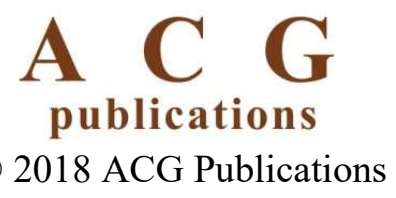

